\title{
INDUCED PLEURIPOTENT STEM CELLS: A PIONEER IN THERAPY
}

\author{
M. Usha Rani ${ }^{1}$, K. S. S. Sarojini Devi², V. Seetharama Raju ${ }^{3}$
}

\section{HOW TO CITE THIS ARTICLE:}

M. Usha Rani, K. S. S. Sarojini Devi, V. Seetharama Raju. "Induced Pleuripotent Stem Cells: A Pioneer in Therapy". Journal of Evolution of Medical and Dental Sciences 2014; Vol. 3, Issue 71, December 18; Page: 15165-15178, DOI: $10.14260 /$ jemds/2014/4038

\begin{abstract}
The pluripotent stem cells can potentially be used to counter a wide range of diseases, from diabetes to spinal cord injury, to childhood leukemia, to heart disease. Human iPS cells are similar to human embryonic stem (ES) cells in terms of proliferation and differentiation ability, and can be generated from adult somatic cells. Now we can easily generate iPS cells from patient's somatic cells and those iPS cells have all genomic information of the patient genome. Many human diseases are caused by genomic mutation. Disease modeling using human iPS cells is newly emerged research field to analyze genetic human diseases. Actually, there are many fatal genetic diseases without effective therapy. To develop newly effective therapies for those diseases, first of all we have to generate disease models. In the past, there had been solely animal models of human genetic disease, such as specific gene knockout mice, transgenic mice and autochthonous diseased animals. Although those models gave us many valuable information regarding the mechanisms of human genetic diseases, most crucial problem is that those models are not human. So it is often difficult to model human diseases using experimental animals. One of the important points is, among humans, each individual shows highly rich in genomic diversity in terms of racial differences and single nucleotide polymorphisms (SNPs). So it has been highly expected to generate not only diseasespecific models, but also disease-specific and patient-specific disease models. To generate patientspecific disease models, now we can use iPS cells. ${ }^{1}$
\end{abstract}

KEYWORDS: Induced pleuripotent Stem Cells, Pioneer, Therapy.

INTRODUCTION: Induced pluripotent stem cells (Also known as iPS cells or iPSCs) are a type of pluripotent stem cell that can be generated directly from adult cells. The iPSC technology was pioneered by Shinya Yamanaka's lab in Kyoto, Japan, who showed in 2006 that the introduction of four specific genes could convert adult cells into pluripotent stem cells. He was awarded the 2012 Nobel Prize along with Sir John Gurdon "for the discovery that mature cells can be reprogrammed to become pluripotent." In November 2007, a milestone was achieved by creating iPSCs from adult human cells; two independent research teams' studies were released - one in Science by James Thomson at University of Wisconsin-Madison and another in Cell by Shinya Yamanaka and colleagues at Kyoto University, Japan. With the same principle used earlier in mouse models, Yamanaka had successfully transformed human fibroblasts into pluripotent stem cells using the same four pivotal genes: Oct3/4, Sox2, Klf4, and c-Myc with a retroviral system. Thomson and colleagues used OCT4, SOX2, NANOG, and a different gene LIN28 using a lentiviral system. ${ }^{2}$

DEFINITION: Pluripotency can be defined in both functional and molecular terms. Functionally, pluripotency refers to the ability of a cell to give rise to cell types of all three germ layers of the embryo: ectoderm, mesoderm and endoderm, as well as the germ line. As such, the very early embryo contains a succession of cells with pluripotent capacity from blastocyst to gastrula stages, but none of 
these cells behave as an ongoing source of stem cells in the later embryo. Embryonic stem (ES) cells are also pluripotent, but exhibit an additional feature: unlimited proliferation in a pluripotent state. Molecular definition of pluripotency requires identification of molecules that support these functional properties. Induced pluripotent stem cells (iPSCs) are adult cells that have been genetically reprogrammed to an embryonic stem cell-like state by being forced to express genes and factors important for maintaining the defining properties of embryonic stem cells. ${ }^{3}$

\section{iPS Cells- Starting cells}

Mouse
Embryonic fibroblasts
Adult tail fibroblasts
Hepatocytes
Gastric epithelial cell
Pancreatic cell
Neural stem cell
B lymphocyte
Keratinocyte

\begin{tabular}{|l|}
\hline \multicolumn{1}{|c|}{ Human } \\
Skin fibroblast \\
Keratinocyte \\
Bone marrow stem cell \\
Peripheral blood cell
\end{tabular}

\section{Transcription factors for reprogramming}

Transcription factors are proteins that bind to DNA and regulafe gene expression

Oct3/4 and Sox2: transcription factors that function in maintaining pluripotency in both early embryos and Es cells.

c-Myc and KIf4: transcription factors that modify chromatin structure so that Oct3 $/ 4$ and Sox 2 can bind to their target: proto-oncogenes 


\section{REVIEW ARTICLE}

Proposed Mechanisms of Reprogramming. ${ }^{4}$

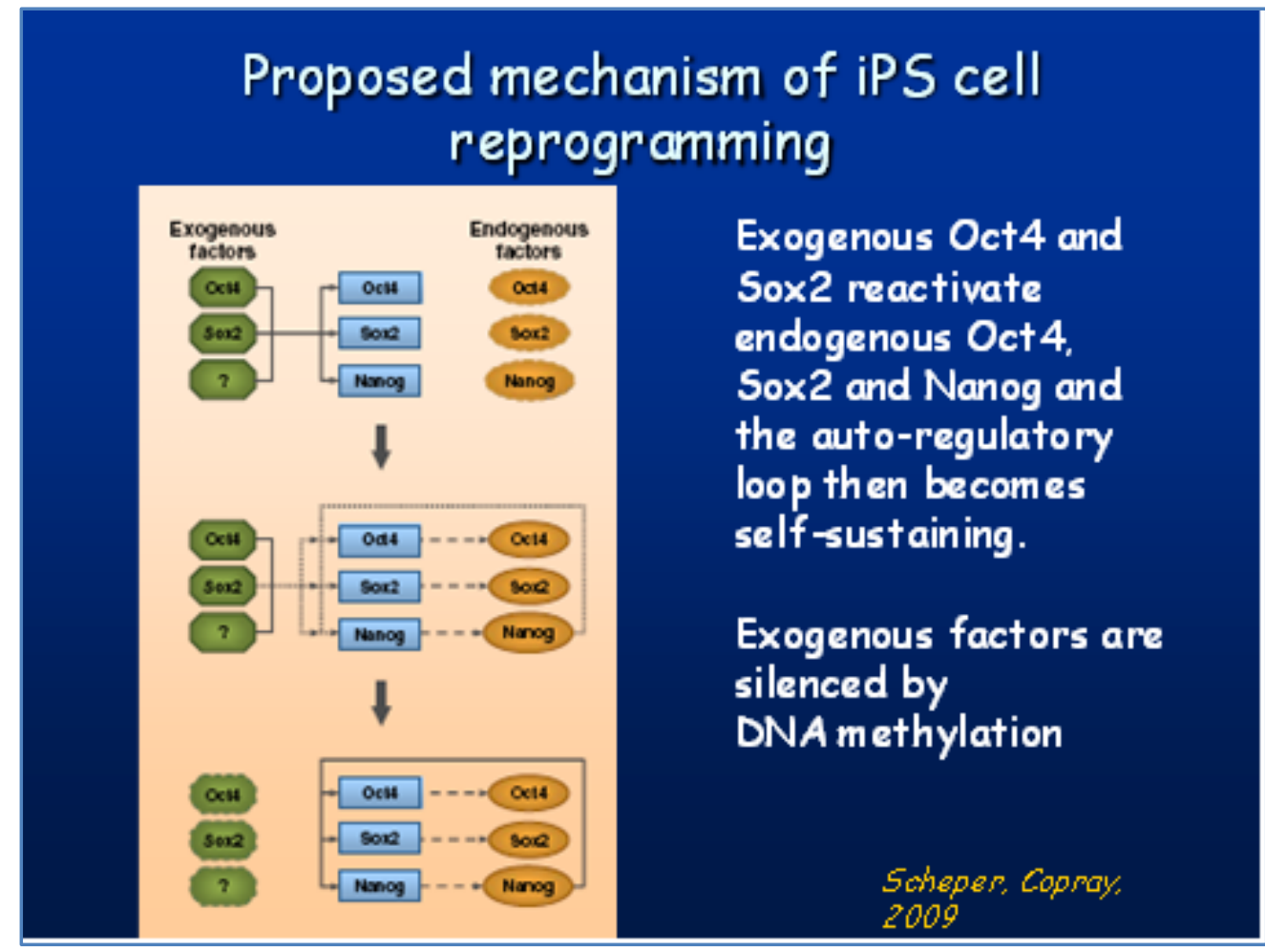

Specific pathways:

TGF- $\beta$ inhibitor replaces Sox 2 and $c M y c$ and induce Nanog (Maherali and Hochedlinger, Curr Biol 2009, Ichida and Eggan 2009) p53 inhibition augments iPS efficiency (Hong and Yamanaka, Nature 2009,Utikal and Hochedlinger Nature 2009, Marion and Blastco Nature 2009, Li and Serrano Nature 2009, Kawamura and Belmonte 2009)

Hypoxia stimulates iPS generation - Yoshida and Yamanaka Cell Stem Cell 2009

Wht signaling stimulates reprogramming efficiency (Marsonm, Jaenisch Cell Stem Cell 2008) 
So to make iPS cells, Yamanaka added four genes to skin cells from a mouse. This started a process inside the cells called reprogramming and within $2-3$ weeks, the skin cells were converted into induced pluripotent stem cells. Scientists can now also do this with human cells, by adding even fewer than four genes. ${ }^{5}$

Human iPS Cells: Human induced pluripotent stem cells (iPSCs, cat\# SC102A-1) were generated by transducing genetically unmodified human fibroblasts with viruses individually encoding the four human transcription factors (Oct4, Sox2, Klf4, and c-Myc) that have been shown to induce the reprogramming of somatic cells to a pluripotent state. The cells were derived using morphological selection criteria and without the use of fluorescent markers or drug selection. When cultured under standard human ES cell culture conditions, the morphology of SBI human iPSCs is identical to that of human ES cells. The cells also express the pluripotency markers Nanog, Oct4, SSEA3 and TRA-1-60, and demonstrate a strong endogenous AP activity. Human iPS cells are adapted to feeder-free conditions and should be grown on Matrigel. Matrigel is available from BD Biosciences. PSGro iPSC Growth Media (SC500M-1) is strongly recommended as the optimal culture medium for those cells. Human iPS cells from SBI are provided at approximately passage 10 and can be passaged 50 times before differentiation. 6,7

\section{Growth Conditions for human iPS Cells:}

Required media and Reagents: nSC500M-1 PSGro Human ESC/iPSC Growth Medium (For feederfree conditions, SC150M-1 CRYO-GOLD Human ESC/iPSC, Cryopreservation Medium, ZRD-Y01/05/25 ROCK Inhibitor (Y-27632). SCR005 Accutase (Millipore), 354277 Matrigel (BD Biosciences)

STORAGE OF IPSCS: Cryopreservation is a critical step in the storage and transportation of human embryonic stem cells (ES cells) and induced pluripotent stem cells (iPS cells). Efficient cryopreservation methods must offer high thawing efficiencies and maintain the pluripotency and differentiation potential of the cells.

Conventional cryopreservation methods for ES cells and iPS cells use fetal bovine serum, which introduces an undefined component into the culture media. $\mathrm{mFreSR}{ }^{\mathrm{TM}}$ is a defined, serum-free cryopreservation medium which allows for rapid recovery following the thawing of ES cells and iPS cells. When used with standard laboratory equipment, $\mathrm{mFreSR}^{\mathrm{TM}}$ allows for successful cryopreservation of ES cells as measured by the undifferentiated, robust subsequent expansion of cells post thaw. Together with $\mathrm{mTeSR}^{\mathrm{TM}} 1$ or $\mathrm{TeSR}^{\mathrm{TM}} 2, \mathrm{mFreSR}^{\mathrm{TM}}$ provides researchers with complete media solutions for maintaining high quality pluripotent cultures and eliminates the use of feeders and serum to reduce variability in experiments. ${ }^{8}$

ES cells cryopreserved in mFreSR ${ }^{\mathrm{TM}}$ have thawing efficiencies 5 to 10 fold higher than conventional thawing methods using serum.

The $\mathrm{mFreSR}^{\mathrm{TM}} 50 \mathrm{~mL}$ bottle can be thawed, dispensed into convenient aliquots then refrozen. Cryopreserved ES cells or iPS cells should be stored at $-150^{\circ} \mathrm{C}$ or in the vapor phase of liquid nitrogen. $\mathrm{mFreSR}{ }^{\mathrm{TM}}$ contains dimethylsulfoxide (DMSO) and is complete and ready-to-use. 


\section{REVIEW ARTICLE}

\section{Advantages of $\mathrm{mFreSR}^{\mathrm{Tm}}$. $^{9}$}

- $\quad$ Easy to use

- Defined and serum-free

- High thawing efficiencies

- Pre-screened components to ensure batch-to-batch consistency.

\section{THERAPEUTIC APPLICATIONS:}

Unique Applications: Each type of pluripotent stem cell has different characteristics that make it useful in different ways, and each has different lessons to teach:

- Induced pluripotent cells (iPS cells) offer a unique chance to model human disease and are already being used to make new discoveries about premature aging, congenital heart disease, cancer and more. As they're made from a person's own cells, they can potentially be manipulated to fix the disease-causing defect and then used to create healthy cells for transplant that won't be rejected by the immune system. Many people also see iPS cells as a positive alternative to pluripotent stem cells from embryos or eggs.

\section{Potentials of iPS cells}

- Ability to differentiate into many cell types

- Easily accessible

- Individual-specific i.e. personalized or non-immunogenic

- Vastly renewable

- Useful for studying mechanisms of disease

- Useful for drug, toxicity testing

Potential application of induced pluripotent stem cells are in cell replacement therapy for Parkinson's disease, Hemophilia, Diabetes mellitus. iPSC have long been used to treat cancers such as leukemia and lymphoma. Pluripotent cells are cells that can become most or all of the 200 cell types of the body, but they cannot become other tissues like placenta. So pluripotent cells, alone without further manipulation, cannot implant and grow into a fetus, baby etc.

Now scientists can take a skin cell, reprogram it and then grow lung tissue, neurons, or other tissue they want to study. Induced pluripotent stem cells are an alternative to cloning because you get stem cells that are a genetic match to a patient, but without creating and destroying a cloned human embryo. Thanks to induced pluripotent stem cell technology, there is hope for those that suffer with CF (Cystic Fibrosis). Advances in basic hepatology have been constrained for many years by the 
inability to culture primary hepatocytes in vitro. But, now, the reprogrammed cells, known as induced pluripotent stem cells (iPSCs), were shown to have the capacity to re-differentiate into almost any human cell type, including hepatocytes.

Three Models of disease treated with iPS-based interventions.

IPS-based therapy.

\section{Disease condition Therapeutic Outcome:}

Sickle cell Disease: Hematopoiesis, functional physiological improvement.

Parkinson's Disease: Dopamine production, symptomatic improvement.

Hemophilia A: Decreased clotting time, survival benefit.

Ischemic heart Disease: Improved cardiac performance.

Translational Perspective: Cardiac dyssynchrony refers to the disparity of wall motion within the heart, a serious consequence of myocardial infarction associated with poor outcome. Scar formation post-infarction compromises device-based pacing, the current standard-of-care for dyssynchronous heart failure. Stem cells are increasingly considered for cardiac repair. However, the impact of stem cell therapy on cardiac dynamics is largely unknown. One study tested the hypothesis that stem cell transplantation could prevent myocardial damage and restore physiological wall motion, achieving cardiac resynchronization. Bioengineered stem cells (i.e. iPS cells) were delivered into acutely infarcted regions in a murine model. High-resolution speckle-tracking echocardiography unmasked global and regional dynamics of cardiac wall motion in vivo, and documented iPS cell-based restoration of synchrony. Compared to progressive dyssynchrony in the absence of stem cell therapy, resynchronized hearts post-iPS cell intervention demonstrated improved electrical conduction and pump function, reduced scar, and reversal of structural remodeling. ${ }^{10,11}$

Tissue Repair: Embryonic cord-blood cells were induced into pluripotent stem cells using plasmid DNA. Using cell surface endothelial/pericytic markers CD31 and CD146, researchers identified 'vascular progenitor', the high-quality, multipotent vascular stem cells. After the iPS cells were injected directly into the vitreous of the damaged retina of mice, the stem cells engrafted into the retina, grow and repaired the vascular vessels.

The recent findings regarding the use of iPSCs for modeling different types of cancer like solid tumors and hematological malignancies represent an ideal tool to study the multiple stages of cancer, for the discovery of new drugs designed for specific biomarkers and for testing drugs' toxicity. Another important point is the possibility to use the iPSCs for immunotherapy in cancer. So, the use of hiPSCs may contribute to the development of future personalized cell therapies and open new possibilities for the treatment of cancer patients. A potential clinical application of hiPSCs in cancer is in the field of immunotherapy. Natural killer (NK) cells play a critical role in host immunity against cancer. In response, cancer develops mechanisms to escape NK cell attack or induce defective NK cells. Current NK cell-based cancer immunotherapy aims to overcome NK cell paralysis using several approaches. One approach is the genetic modification of fresh NK cells or NK cell lines to highly express cytokines, Fc receptors, and/or chimeric tumor-antigen receptors. Therapeutic NK cells can be derived from various sources, including peripheral or cord blood cells, stem cells, or even induced pluripotent stem cells (iPSCs) and a variety of stimulators can be used for large scale production in laboratories or good manufacturing practice. 


\section{REVIEW ARTICLE}

Potential applications of human iPSCs: The iPSCs technology can be potentially used in disease modeling, drug discovery, gene therapy and cell replacement therapy. Differentiated cells are acquired by biopsies from human tissues and in vitro cultured under stem cell transcription factors, such as SOX2 (SRY-box containing gene 2), c-Myc (v-myc avian myelocytomatosis viral oncogene homolog), OCT4 (octamer-binding transcription factor 3), and KlF4 (Kruppel-like factor 4). After induction of pluripotency phenotype, the cells, known as iPSCs, can be utilized for re differentiation in specific disease, to drug screening, or to have the genomic defect corrected, and then the iPSCs become able to be reutilized as health cells in the regenerative therapies. ${ }^{12,13}$

It is generally accepted that in vitro reprogramming of somatic cells into induced pluripotent stem cells offers new applications in basic research, diagnosis and cell therapy. The most attractive application would be the production of patient-specific donor cells for cell replacement and/or tissue substitution. For example, skin fibroblast-derived iPS cells showed the potential to differentiate into islet-like clusters and to release insulin in response to glucose thus potentially providing a strategy for the treatment of diabetes (Tateishi et al., 2008). ${ }^{14}$

Another exciting strategy may be the combination of iPS cell generation combined with somatic gene therapy similarly as shown for mouse cells by Jaenisch and coworkers (Hanna et al., 2007).

However, other applications of iPS cells are also feasible, such as the use of iPS cells to study pathomechanisms of diseases, including Parkinson's disease and Huntington's disease, juvenile-onset type 1 diabetes mellitus or amyotrophic lateral sclerosis (Dimos et al, 2008; Park et al., 2008). Recently, skin fibroblasts from a patient with spinal muscular atrophy were reprogrammed into proliferative iPS cells that maintained the disease genotype and were able to differentiate into motor neurons. The iPS cells showed selective deficits typical for the disease as well as drug responsiveness (Ebert et al, 2009). This example demonstrates that iPS cells may be a promising tool to study the mechanisms of diseases and the effects of drugs thus enabling the development of new therapies. Moreover, similarly to human ES cells, iPS cells could be applied in toxicology and pharmacology. For instance, iPS cells differentiating in vitro may be used as an alternative system for screening of embryotoxic and/or teratogenic substances (Caspi et al., 2008).

Although much additional basic research will be required before iPSCs can be applied in the clinic, these cells represent multi-purpose tools for medical research. Using the techniques described in this article, researchers are now generating myriad disease-specific iPSCs. For example, dermal fibroblasts and bone marrow-derived mesencyhmal cells have been used to establish iPSCs from patients with a variety of diseases, including ALS, adenosine deaminase deficiency-related severe combined immunodeficiency, Shwachman- Bodian-Diamond syndrome, Gaucher disease type III, Duchenne and Becker muscular dystrophies, Parkinson's disease, Huntington's disease, type 1 diabetes mellitus, Down syndrome/trisomy 21 and spinal muscular atrophy. iPSCs created from patients diagnosed with a specific genetically-inherited disease can then be used to model disease pathology. For example, iPSCs created from skin fibroblasts taken from a child with spinal muscular atrophy were used to generate motor neurons that showed selective deficits compared to those derived from the child's unaffected mother.As iPSCs illuminate the development of normal and disease-specific pathologic tissues, it is expected that discoveries made using these cells will inform future drug development or other therapeutic interventions. 
One particularly appealing aspect of iPSCs is that, in theory, they can be directed to differentiate into a specified lineage that will support treatment or tissue regeneration. Thus, somatic cells from a patient with cardiovascular disease could be used to generate iPSCs that could then be directed to give rise to functional adult cardiac muscle cells (cardiomyocytes) that replace diseased heart tissue, and so forth. Yet while iPSCs have great potential as sources of adult mature cells, much remains to be learned about the processes by which these cells differentiate. For example, iPSCs created from human and murine fibroblasts can give rise to functional cardiomyocytes that display hallmark cardiac action potentials. However, the maturation process into cardiomyocytes is impaired when iPSCs are used-cardiac development of iPSCs is delayed compared to that seen with cardiomyocytes derived from ESCs or fetal tissue. Furthermore, variation exists in the expression of genetic markers in the iPSC-derived cardiac cells as compared to that seen in ESC-derived cardiomyocytes. Therefore, iPSC-derived cardiomyocytes demonstrate normal commitment but impaired maturation. An intriguing phenomenon unique to human pluripotent stem cells is dissociation-induced apoptosis, which has been a technical problem for various cellular manipulations. The discovery that this apoptosis is suppressed by ROCK inhibitors brought revolutionary change to this troublesome situation.

Induced Pluripotent Stem Cells for Tissue Regeneration: Induced pluripotent stem cells (iPSCs) have tremendous potential for patient-specific cell therapies, which bypasses immune rejection issues and ethical concerns for embryonic stem cells. IPSC-derived neural crest stem cells (NCSCs) can differentiate into a variety of cell types, and hold promise for the therapies of diseases such as nerve injuries, demyelinating diseases, spina bifida, vascular diseases, osteoporosis and arthritis. Stem cell therapies can accelerate nerve regeneration and avoid the degeneration of muscle and other tissues lack of innervation. Since iPSC-NCSCs can promote the myelination of axons, the therapies for nerve injuries could also be adopted to treat demyelinating diseases. $\mathbf{1 5}$

Future applications and challenges for iPS Cells: Reprogramming holds great potential for new medical applications, such as cell replacement therapies. Since iPS cells can be made from a patient's own skin, they could be used to grow specialized cells that exactly match the patient and would not be rejected by the immune system. If the patient has a genetic disease, the genetic problem could be corrected in their iPS cells in the laboratory, and these repaired iPS cells used to produce a patientspecific batch of healthy specialized cells for transplantation. But this benefit remains theoretical for now.

Until recently, making iPS cells involved permanent genetic changes inside the cell, which can cause tumours to form. Scientists have now developed methods for making iPS cells without this genetic modification. These new techniques are an important step towards making iPS-derived specialized cells that would be safe for use in patients. Further research is now needed to understand fully how reprogramming works and how iPS cells can be controlled and produced consistently enough to meet the high quality and safety requirements for use in the clinic. Applications in basic biology, as well as cellular therapeutics and disease modeling, combined with other emerging technologies, such as epigenomic profiling and deep sequencing, we predict a bright future for iPSC technology in both the clinic and the laboratory. 
The transplant Process: There are several steps in the transplant process. The steps are much the same, no matter what type of transplant you are going to have.

Patient evaluation and Preparation: Patient will first be evaluated to find out if he/she is eligible for a transplant.

\section{MEDICAL TESTS:}

- Many different medical tests may be done, and questions will be asked to try to find out how well you can handle the transplant process. These might include:

- HLA tissue typing.

\section{A complete health history and physical Exam: ${ }^{16}$}

- Evaluation of psychological and emotional strengths.

- Identifying who will be the primary caregiver throughout the transplant process.

- Bone marrow biopsy.

- CT (Computed tomography) scan or MRI (Magnetic resonance imaging).

- Heart tests, such as an EKG (Electrocardiogram) or echocardiogram.

- Lung studies, such as a chest x-ray and PFTs (Pulmonary function tests).

- Consults with other members of the transplant team, such as a dentist, dietitian, or social worker.

- Blood tests, such as a complete blood count, blood chemistries, and screening for viruses like hepatitis B, CMV and HIV.

\section{HOSPITAL ADMISSION:}

\section{Conditioning Treatment:}

- Conditioning, also known as bone marrow preparation or myeloablation, is treatment with high-dose chemo and/or radiation therapy. It's done for one or more of these reasons:

- To make room in the bone marrow for the transplanted stem cells

- To suppress the patient's immune system to lessen the chance of graft rejection

- To destroy all of the cancer cells anywhere in the patient's body

- Conditioning treatment is not same for all transplants. The treatment will be planned and based on the type of cancer patient has, the type of transplant, and any chemo or radiation therapy the patient had in the past.

- If chemo is part of the treatment plan, it will be given in an intravenous (IV) line or as pills. If radiation therapy is planned, it's given to the entire body (called total body irradiation or TBI). TBI may be given in a single treatment session or in divided doses over a few days.

\section{INFUSION OF STEM CELLS:17,18}

- After the conditioning treatment, patient is given a couple of days to rest before getting the stem cells. They will be given through the IV catheter, much like a blood transfusion. If the stem cells were frozen, patient may be given some medicines before the stem cells are given. This is done to reduce the risk of reacting to the preservatives that are used when freezing the cells. 


\section{REVIEW ARTICLE}

- If the stem cells were frozen, they are thawed in warm water then given right away. For allogeneic or syngeneic transplants, the donor cells may be harvested in an operating room, and then processed in the lab. Once they are ready, the cells are brought in and infused. The length of time it takes to get all the stem cells depends on how much fluid the stem cells are in.

- This is big step and often has great meaning for recipients and their families. Many people consider this their rebirth or chance at a second life. They may celebrate this day as they would their actual birthday.

\section{DISCHARGE:}

\section{Discharge Planning:}

- The discharge process actually begins weeks before the transplant. It starts with the transplant team teaching the patient and his primary (main) caregiver about:

- The precautions that will need to take.

- How to prepare patient's home.

- How to care for the central venous catheter.

- How to take good care of mouth and teeth.

- What foods patient should and shouldn't eat.

- Activities the patient can and can't do.

- When to call the transplant team or other health care professionals.

- Who will take the role of primary caregiver and what the job will involve, and who will be the back-up caregiver in case the main caregiver gets sick and can't be near you.

\section{Discharge Criteria:}

- For the most part, transplant centers don't discharge patients until they meet the following criteria:

- They have no fever for 48 hours.

- They are able to take and keep down pills or other drugs for 48 hours.

- Their nausea, vomiting, and diarrhea are controlled with medicine.

- Their neutrophil count (absolute neutrophil count or ANC) is at least 500 to $1,000 / \mathrm{mm}^{3}$.

- They have a hematocrit of at least $25 \%$ to $30 \%$.

- They have a platelet count of at least 15,000 to $20,000 / \mathrm{mm}^{3}$.

- They have someone to help them at home and a safe and supportive home environment. If patients do not meet all of these requirements, but still don't need the intensive care of the transplant unit, they may be moved another oncology unit.

\section{REHABILITATION:}

- The roller coaster ride often continues after the patient goes home. Plus, he will be feeling pretty tired after going through the transplant process. After discharge, in the rehabilitation period, some people have physical or mental health problems. These ongoing needs must now be managed at home and caregiver and friend/family support is very important.

- Transplant patients are still followed closely during rehab. He may need daily or weekly exams along with things like blood tests, chest x-rays, bone marrow tests, or spinal taps (lumbar punctures). During early rehab, he also might need blood and platelet transfusions, antibiotics, 


\section{REVIEW ARTICLE}

or other treatments. The exams are frequent at first, maybe even every day, but will be needed less often if things are going well. It can take 6 to 12 months, or even longer, for blood counts to get close to normal and the immune system to work well.

\section{Adult Stem Cell Transplantation Process. 19,17,18,16}

\section{The Adult stem cell transplant Process.}

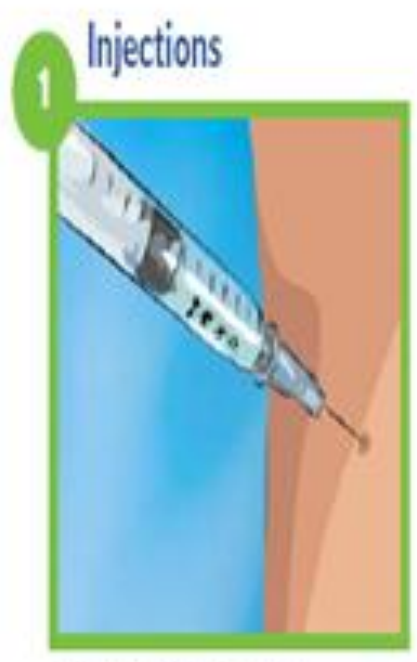

liedons d moliston agats

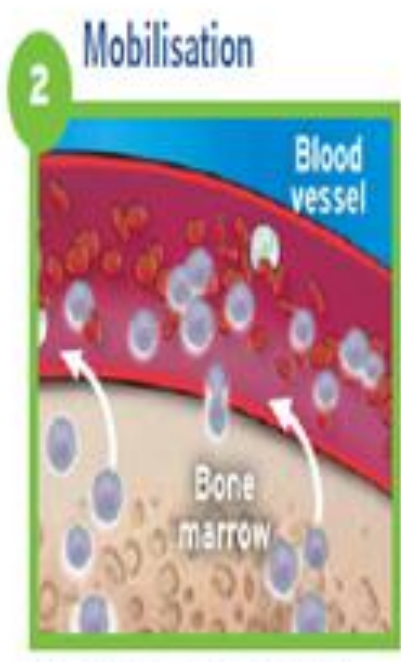

Sen clis $x$ e dindted to nov ath te

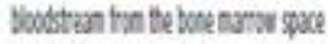

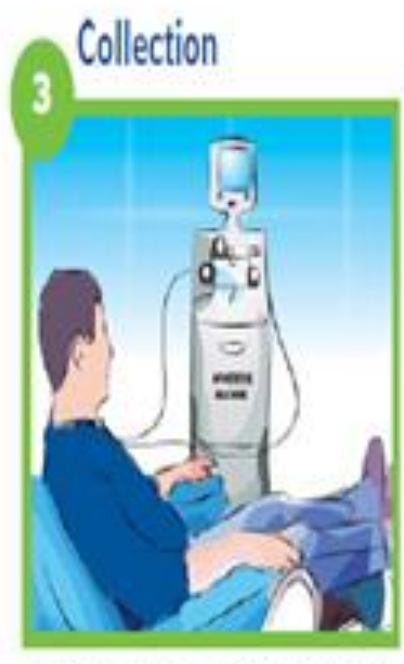

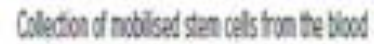
wingte yetess notive 


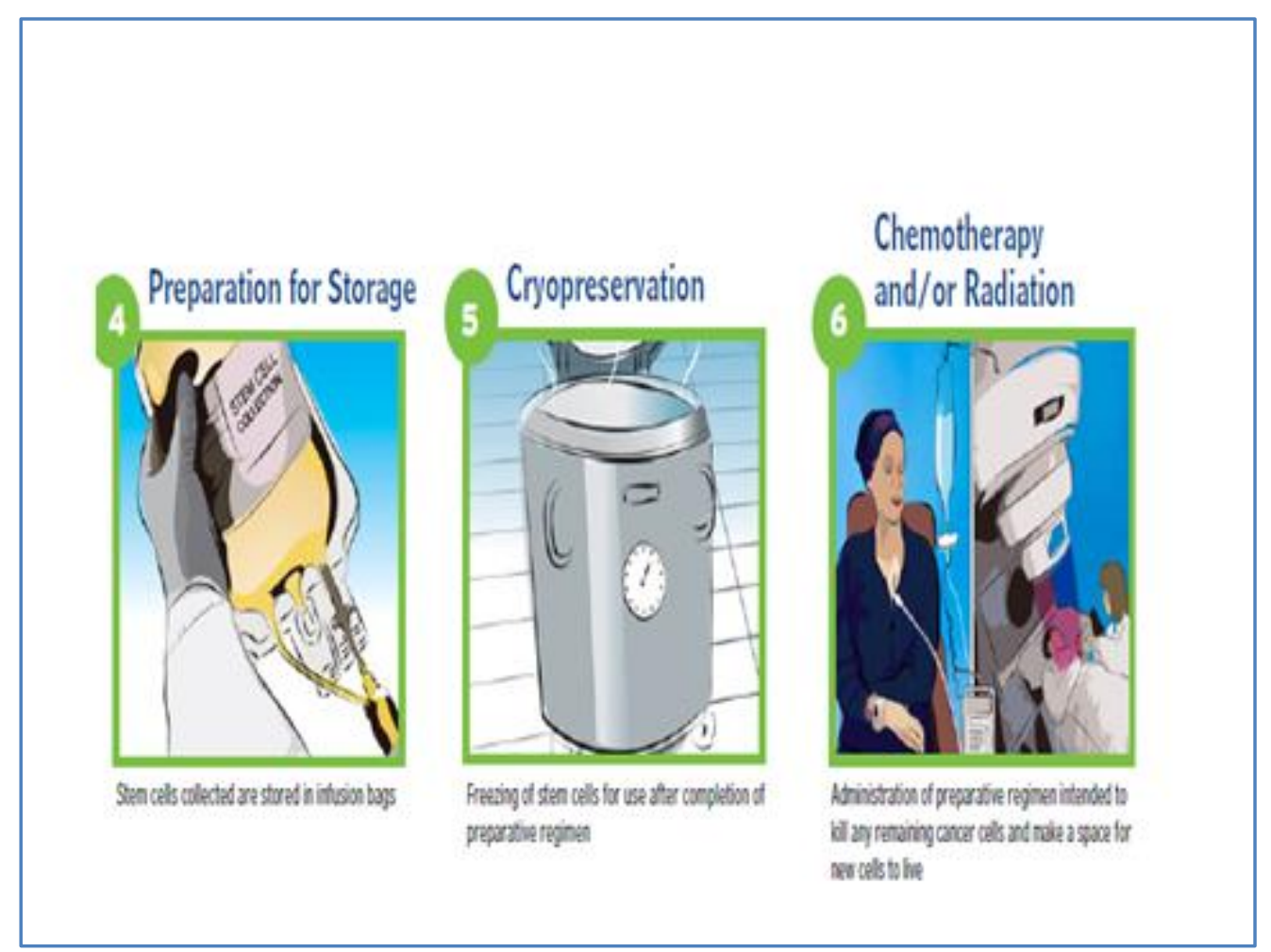




\section{REVIEW ARTICLE}

\section{REFERENCES:}

1. Casimir de Rham and Jean Villard: Review article: Potential and Limitation of HLA-Based Banking of Human Pluripotent Stem Cells for Cell Therapy. Journal of Immunology Research Volume 2014 (2014), Article ID 518135.

2. Craig J. Taylor, Eleanor M. Bolton and J. Andrew Bradley::pluripotent stem cell banking Phil. Trans. R. Soc. B 2011 366, doi: 10.1098/rstb.2011.0030, published 4 July 2011.

3. Matthias Stadtfeld1, and Konrad Hochedlinger: Induced pluripotency: history, mechanisms, and applications.-doi: 10.1101/gad.1963910 Genes \& Dev. 2010. 24: 2239-2263.

4. Ali Nsair e, Katja Schenke-Layland, Ben Van Handel, Denis Evseenko, Michael Kahn, Peng Zhao, et al. Characterization and Therapeutic Potential of Induced Pluripotent Stem Cell-Derived Cardiovascular Progenitor Cells: Published: October 09, 2012, DOI:10.1371/journal.pone.0045603

5. Michael Peitz and Annette Pusch: Reprogramming: how to turn any cell of the body into a pluripotent stem cell. -taken from http://www.eurostemcell.org/factsheet/ips-cells-andreprogramming-turn-any-cell-body-stem-cell

6. Teresa de Souza Fernandez, Cecilia de Souza Fernandez, and André Luiz Mencalha Human Induced Pluripotent Stem Cells from Basic Research to Potential Clinical Applications in CancerBio Med Research International Volume 2013 (2013), Article ID 430290, web source http://dx.doi.org/10.1155/2013/430290.

7. Induced pluripotent stem cell From Wikipedia, the free encyclopedia.

8. Sachiko Yamasaki, Yuki Taguchi, Akira Shimamoto, Hanae Mukasa, Hidetoshi Tahara, Tetsuji Okamoto mail Generation of Human Induced Pluripotent Stem (iPS) Cells in Serum- and FeederFree Defined Culture and TGF- $\beta 1$ Regulation of Pluripotency -Published: January 29, 2014, DOI: 10.1371 /journal.pone.008715.

9. Embryonic stem cells \& iPS cells (Human) taken from http://www.stemcell.com/en/Products/Cell-type/Embryonic-stem-cells-iPS-cells-Human.aspx

10. Zhiqiang Liu, Jin Zhou, Haibin Wang, Mengge Zhao and Changyong Wang: Current status of induced pluripotent stem cells in cardiac tissue regeneration and engineering. Regenerative Medicine Research 2013, 1:6 available from http://www.regenmedres.com/content/1/1/6

11. Satsuki Yamada, Timothy J. Nelson, Garvan C. Kane, Almudena Martinez-Fernandez et al. Induced pluripotent stem cell intervention rescues ventricular wall motion disparity, achieving biological cardiac resynchronization post-infarction-doi: 10.1113 / jphysiol. 2013. 252288 September 1, 2013 the Journal of Physiology, 591, 4335-4349.

12. ATCC-DYR0100 Human Induced Pluripotent Stem Cells: taken from http://www.atcc.org/en/Global/Products/6/B/6/4/ACS-1011.aspx

13. Konrad Hochedlinger: presented in cme winter symposium 12 at Department of Stem Cell and Regenerative Biology Howard Hughes Medical Institute, Harvard Stem Cell Institute.

14. Anahita Shaer, ${ }^{1,2}$ Negar Azarpira,2 Akbar Vahdati, ${ }^{2}$ Mohammad Hosein Karimi, ${ }^{2}$ Mehrdad Shariati $^{3}$ Differentiation of Human-Induced Pluripotent Stem Cells Into Insulin-Producing Clusters. EPUB DOI: 10.6002/ect.2013.0131

15. Chen LW, Kuang F, Wei LC, Ding YX, Yung KK, Chan YS: Potential application of induced pluripotent stem cells in cell replacement therapy for Parkinson's disease. CNS Neurol Disord Drug Targets. 2011 Jun; 10 (4): 449-58. 


\section{REVIEW ARTICLE}

16. Hooper PJ, Santas EJ. Peripheral blood stem cell transplantation. Oncol Nurs Forum. 1993; 20 (8): quiz 1222-3.

17. Kapustay PM. Blood cell transplantation: concepts and concerns. Semin Oncol Nurs. 1997; 13 (3):151-163.

18. National Cancer Institute. Bone marrow transplantation and peripheral blood stem cell transplantation fact sheet. Available at http://cancer.gov/cancertopics/factsheet/Therapy/bone-marrow-transplant Accessed July 7, 2009.

19. Walker F, Roethke SK, Martin G. An overview of the rationale, process, and nursing implications of peripheral blood stem cell transplantation. Cancer Nurs. 1994; 17(2):141-148.

\section{AUTHORS:}

1. M. Usha Rani

2. K. S. S. Sarojini Devi

3. V. Seetharama Raju

\section{PARTICULARS OF CONTRIBUTORS:}

1. Associate Professor, Department of Physiology, Andhra Medical College, Visakhapatnam.

2. Associate Professor, Department of Physiology, Andhra Medical College, Visakhapatnam.

3. Assistant Professor, Department of Physiology, Andhra Medical College, Visakhapatnam.

\section{NAME ADDRESS EMAIL ID OF THE CORRESPONDING AUTHOR:}

Dr. M. Usha Rani,

Flat No. 407,

Sai Towers, Rednam Gardens,

Visakhapatnam-530002.

Email: ushaprasad6@gmail.com

Date of Submission: 02/11/2014.

Date of Peer Review: 03/11/2014.

Date of Acceptance: 10/11/2014.

Date of Publishing: 17/12/2014. 